\title{
AN RFID BASED DEVICE FOR INNOVATING THE PATIENTS MANAGEMENT
}

\section{GIORGIO A.}

Politecnico di Bari, Dipartimento di Elettrotecnica ed Elettronica, Via E. Orabona 470125 Bari, ITALY. *Corresponding Author: Email- a.giorgio@poliba.it

Received: December 07, 2011; Accepted: March 15, 2012

\begin{abstract}
The advent of computer systems in support of medical procedures has significantly improved healthcare services quality, and the patients' general quality of life. Nevertheless, these benefits not always improve patient's health directly. For example, in the case of an emergency relief to an injured or sick person, it is essential to obtain timely patient's past medical history in order to prevent the supply of incorrect treatments which could further aggravate the situation. In this context, the designed system wants to demonstrate how to dramatically improve medical history acquisition procedures and face the most serious situations, using RFID technology; not forgetting, among others, important aspects as integration into an existing computer system, or the economic factor.
\end{abstract}

Key words- RFID technology, Patients Management, CT, MRI, PET

Citation: Giorgio A. (2012) An RFID Based Device for Innovating the Patients Management. International Journal of Bioinformatics Research, ISSN: 0975-3087 \& E-ISSN: 0975-9115, Volume 4, Issue 1, 2012, pp.-254-257.

Copyright: Copyright@2012 Giorgio A. This is an open-access article distributed under the terms of the Creative Commons Attribution License, which permits unrestricted use, distribution, and reproduction in any medium, provided the original author and source are credited.

\section{Introduction}

The effect of information and automation technology is manifesting more and more widely in medical procedures, bringing substantial benefits to the health and welfare of all patients. We can think about already existing applications as the ability to book a medical examination via internet, or use sophisticated diagnostic techniques (CT, MRI, PET), or the possibility to perform minimally invasive endoscopic techniques, or telemedicine.

Among these, data processing certainly is a crucial aspect for patient care. Health management and control, indeed, are based on using, transmitting and comparing a large amount of data, information and heterogeneous knowledge. However, in recent years the need to exchange data has increased dramatically, both within a health facility (among different subjects and specialized units), and among geographically distant facilities. Amongst other things, with the rising organization cost and complexity, we can't think to disregard an adequate information system consisting of management software and complex databases which ensure organization control and optimization [1].
Although computerization drastically increases the efficiency and effectiveness of data processing procedures, has less impact in terms of increasing the care quality or the patients' quality of life, especially for elderly, chronically ill, accident-prone patients.

In order to address this shortcoming, we developed a system based on RFID (Radio Frequency IDentification) consisting on both hardware and software, that would allow to improve the health care quality by improving the patients management and that would to be perfectly integrated into any local / national or international health information system and data base [2]. In fact, as will be deeply explained in following sections, by equipping any person of an RFID smart card, and by equipping any hospital of an RFID wireless smart card reader for patient identification connected to a computer, we have implemented the instantaneous and automatic access to all patient's clinical / medical history information stored in a personal folder of files into database containing one folder for each patient. The database should be world wide and everywhere located, not necessarily on the hospital computer, if the hospital computer is internet connected. 
The importance of having the patient's medical history is also crucial to speed up healing process and reduce risk likelihood. Consider, for example, a patient must be urgently admitted to hospital and does not know or can not report all the critical information needed before surgery, he should be first subjected to a series of investigations and then undergo surgery, or suffer intervention without collecting the above information with high risk of mortality. The designed system meets requirements and benefits ranging from simple procedures streamlining for acquisition of individual's clinical data, to the access to all clinical / history data for patients unable to exhibit them (because they are unconscious, or because don't remember, etc.), to the automatic creation of a database in compliance with health service computerization requirements.

Here we will discuss the support technologies and the device designed characteristics in particular.

\section{RFID Technology: a quick reminder \\ General Information}

RFID (Radio Frequency Identification) is a wireless technology that represents an innovative solution in the field of processes automation. Although its origin is not recent, it will provoke a veritable revolution in every productive sector in the coming years.

The reason why only in recent years RFID has spread, is because its use has changed over times

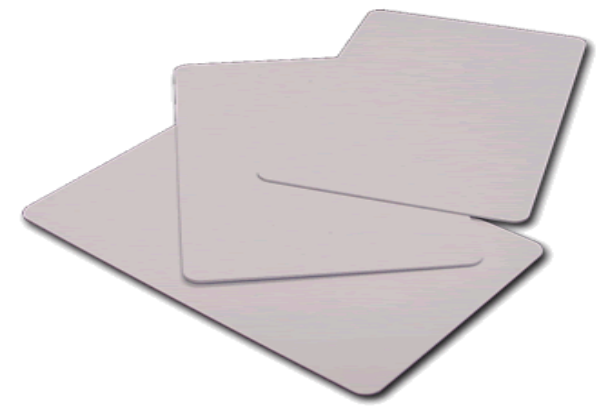

Fig. 1- Example of RFID card transponder used in our project. Operating frequency at 134,2 Khz; ISO/IEC11784/11785 compliant

and, if until recently, RFID could be considered as a still evolving technology, currently is moving towards a stage of expression maturity.

The very acronym definition is very clear in specifying and limiting the technology involved:

- Is a technology that allows the identification (i.e. the unique recognition.) of an object or living being.

- Is a technology that uses radio frequency.

At the basis of its operation there is an intuitive idea of being able to identify, through an intelligent label (called "tag", "transponder" or "data-carrier device") without any need for physical connection, any object such as products, animal or people. These transponders, which example is shown in fig. 1, unlike their predecessors bar codes, have the ability to store on a chip an information that can be transmitted via radio waves to the appropriate reading devices (readers or interrogators). Then data is sent to a central computer to be interpreted and processed [2].

To understand the significance of this mechanism is enough to think that on a single chip you can put several pieces of infor- mation (from the serial identification code, name, last name, etc.) and that the readers, depending on applications, are able to capture tags information at a distance ranging from a few centimeters to several meters.

\section{RFID Technology in Designed System}

The best RFID transponder package matching the project criteria is compliant to the ISO 7816 standard, where we find credit cards, personal identification cards and especially smart cards. In fact, these are often used when transactions must be processed quickly or hands-free, such as on mass transit systems, where a smart card can be used without even removing it from a wallet.

RFID technology has a very large presence in the smart cards field. There are standards like ISO/IEC 14443 or ISO/IEC 15693 that allow for communications at distance ranging from a few centimeters up to a meter at operative frequency of $13,56 \mathrm{Mhz}$, or also ISO/IEC 11784 ISO/IEC 11785 allow for communication at $134,2 \mathrm{Khz}$ frequency and the just mentioned distance.

Smart cards are advertised as suitable for personal identification tasks, because they are engineered to be tamper resistant. The embedded chip of several smart card models usually implements some cryptographic algorithm.

Last but not least, the amount of embedded memory varies from a few bits up to a megabyte and allows us to insert all necessary personal and clinical data. Table I shows what kind of information we can eventually store in a smart card transponder, and which data are stored in the tags used in this project.

\section{Table 1- Data Stored in Transponder Memory}

\begin{tabular}{|c|c|c|c|}
\hline Data type & $\begin{array}{l}\text { Texas Instruments } \\
\text { RI-TRP-R4FF } \\
\text { Memory: } 80 \text { bit } \\
\text { (Used in the project) }\end{array}$ & $\begin{array}{l}\text { Texas Instruments } \\
\text { RI-TRP-DR2B } \\
\text { Memory: } 1360 \text { bit } \\
\text { (Used in the project) }\end{array}$ & $\begin{array}{l}\text { Mifare } \\
\text { SmartMX } \\
\text { Memory: 20KB to } \\
\text { 144KB (example) }\end{array}$ \\
\hline Tag Code & $x$ & $x$ & $x$ \\
\hline Italian Fiscal Code & & $x$ & $x$ \\
\hline Name & & $x$ & $x$ \\
\hline Address & & $x$ & $x$ \\
\hline Other personal data & & $x$ & $x$ \\
\hline Medical History & & & $x$ \\
\hline Tests & & & $x$ \\
\hline Therapies & & & $x$ \\
\hline Allergies & & & $X$ \\
\hline Other Clinical Data & & & $x$ \\
\hline
\end{tabular}

\section{Designed System Features and Functionality}

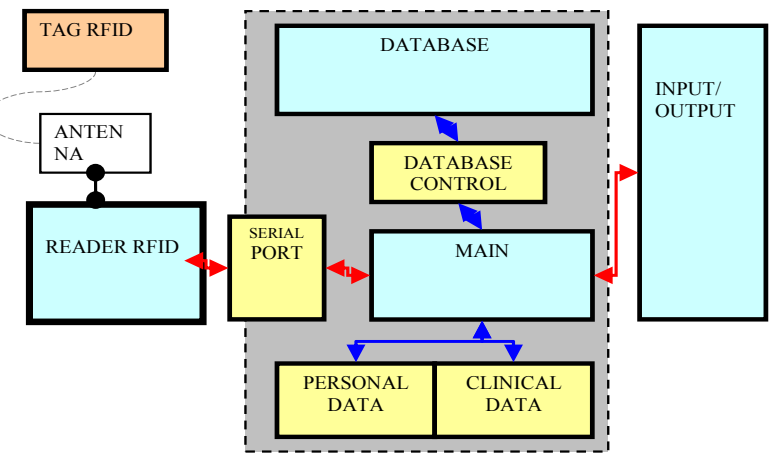

Fig. 2- System block diagram. Note that blue background blocks are the main constituents of the project, instead yellow background ones handle data processing. In red the main path of RFID data. 
The draft provides that any health facility user, who previously authorises the processing of his personal details, is supplied with an RFID tag card, which stores his clinical and personal data.

Then, the designed system allows doctors a very quick consulting of the clinical data of a patient; moreover, the system is perfectly integrated into any local/national or international health information system and data base.

The importance of having promptly the patient's medical history is also crucial to speed up healing process and reduce risk likelihood. Consider, for example, a patient must be urgently admitted to hospital and does not know or cannot report all the critical information needed before surgery, he should be first subjected to a series of investigations and then undergo surgery, or suffer intervention without collecting the above information with high risk of mortality.

Therefore, by equipping any person of an RFID smart card and by equipping any hospital of an RFID wireless smart card reader for patient identification connected to a computer, we have implemented the instantaneous and automatic access to all patient's clinical/medical history information stored in a personal folder of files into database containing one folder for each patient.

The database should be world wide and everywhere located, not necessarily on the hospital computer, if the hospital computer is internet connected.

The designed system meets requirements and benefits ranging from simple procedures streamlining for acquisition of individual's clinical data, to the access to all clinical / history data for patients unable to exhibit them (because they are unconscious, or because don't remember, etc.), to the automatic creation of a database in compliance with health service computerization requirements.

Assuming the use of the system in the hospital or nursing home reception, if the user comes with wearing the tag within the detection distance (less than a meter.), the system initiates a series of automatic procedures for the unique recognition of the individual and the search of his data within the central database, which, then, are also printed on the screen of an operator.

If a detected tag contains invalid reference data in the database, or if the patient is inserted for the first time in the database, it is quite automatically updated by the system by creating, without any human intervention, a new record that contains the data taken directly from the tag and possibly displaying the new acquired information. Then, for the new patient, the system provides the automatic creation of the personal folder as the patient has been identified by the RFID reader, and the automatic transfer of the main data from the personal smart card (self-creating data base). Therefore, the system allows you to create a dynamic database that updates itself without the aid of any employee and that makes immediately available to health professionals (doctors, paramedics, etc..) all information relating to patient data stored in the its own RFID smart card, such as medical history including current therapies, diseases, examinations reports, specialist reports and all that is deemed useful to include in the data base. The remarkable feature is that the access to the patient's folder occurs suddenly and automatically on a computer connected to the RFID reader, as the patient has been identified. In this way is not needed for any description by the same patient about its own clinical history thus reducing drastically the misunderstanding and the forgetfulness.
To fulfill these tasks, the system was modeled according to the block diagram in fig 2.

As previously mentioned, the system architecture consists of a mixed hardware and software. The hardware component is represented by a RFID technology kit including smart cards (tags) and a receiver (reader). Their radio frequency interaction allows the unambiguous people recognition.

The software, however, developed in Visual Studio, consists primarily of a relational database simulating a health facility database with patients' clinical data inside, and secondly of a very intuitive graphic interface to manage both the RFID device and the data base.

The scheme in fig. 2 gives a good idea how the system is achieved. From the left, we have the presence of the RFID tag assigned to each patient which interfaces to the reader through the appropriate antenna; the RFID reader, in turn, is connected via serial cable to the port available on the PC and controlled by a dedicated software class. The data flow goes directly into the main form, which provides data management and visualization through the dedicated forms. Finally, the same core module provides the graphical interface for input / output with the healthcare professional.

The software interface is designed to provide maximum ease of use and maximum usability of the information. In fact, the medical staff, excluding some self-taught, did not usually experience with the computing device. So this requires a rational approach that privileges the automatic rather than total control of the instrument but without sacrificing functionality.

In the main window (Fig. 3.) are condensed all necessary controls to access software features as the data table and controls for managing records and information related to them. These controls reproduce features like search, delete, edit, create new record, regardless of the particular format of the database connected to the system (Access, SQLServer, MySQL). Indeed, designing the database control class through ADO.NET methods and the SQL language, allows a certain independence from the data source, which, in this context, translates into a great ease of integration with existing databases [3].

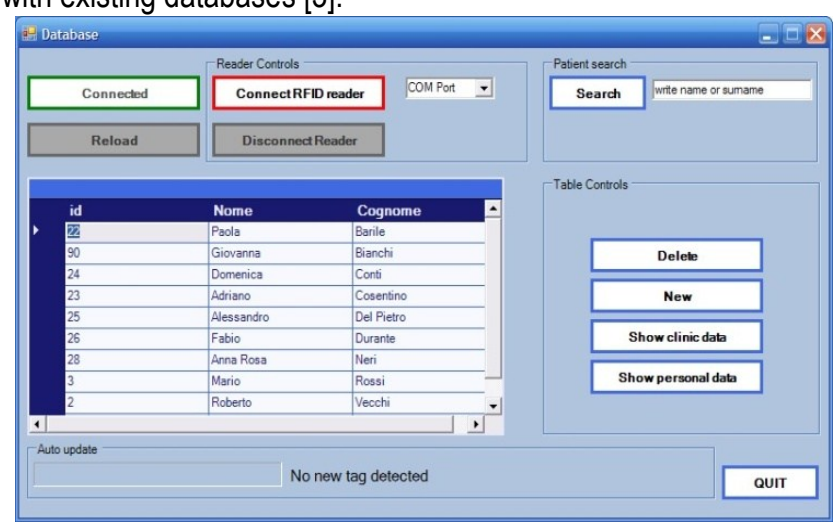

Fig. 3- Main menu

In addition there are buttons for connecting and disconnecting the RFID device from database.

Buttons for RFID reader are only two, allowing the connection and disconnection from the device. The procedures for reading the tags, recognizing and displaying information, are autonomously managed by the software, which requires the reader to make cy- 
clical readings every two seconds. The device responds in three main ways:

1. Tag not found

2. Tag found

3. Tag is invalid o not recognized

An answer like "tag not found" occurs in the absence of a transponder within the operative range. A message such as "tag detected", however, starts procedures for the recognition and eventual data drawing, which takes about one second of operation. The third message ("Invalid tag or not recognized") involves the display of warning messages in circumstances of incorrectly coded or partially damaged tags.

Even if reader detects a valid tag that does not match any reference in the database, or if the patient is included for the first time in the database, he is quite automatically updated by the system by creating, without any human intervention, a new record that contains the data taken directly from the tag and possibly displaying the new acquired information. Then, for the new patient, the designed system provides the automatic creation of the personal folder as the patient has been identified by the RFID reader, and the automatic transfer of the main data from the personal smart card. This important feature allows you to create a dynamic database that updates itself without the aid of any employee and that makes immediately available to health professionals (doctors, paramedics, etc.) all information relating to patient data, medical history, including current therapies, diseases, examinations reports, specialist reports and all that is deemed useful to include in the data base.

Moreover, the speed of data exchange with the effectiveness of a good RFID system spares the transponder owner to show his card, and linger near the recognition point, in the interest of timely intervention in urgent cases.

Finally, we should highlight that the system also has the possibility of writing information on transponder, so any new intervention, examination or, a more general clinical and medical history data updating can be always recorded on the patient smart card.

\section{Conclusions}

The use of well designed system actually allows a drastic reduction in recognition time and the immediate availability of patients' generic and clinical data. For example, in the case of manual recognition and data acquisition, the time ranges from a minimum of a few minutes to a maximum of about 40 minutes, while exploiting the automation of our system, we need about one or two seconds. It's obvious that in a urgency situation, the immediate availability of the subject's medical history provides the choice of an effective and especially timely cure.

Another remarkable feature is that the access to the patient's folder or the creation of it occurs suddenly and automatically on a computer connected to the RFID reader, as the patient has been identified. In this way is not needed for any description by the same patient about its own clinical history thus reducing drastically the misunderstanding and the forgetfulness.

Furthermore, the particular system architecture with the extreme simplicity and high automation, do not steal time to health personnel to assimilate new procedures, contributing to an effective integration in any existing health facility information systems.
Also the economic sphere should not be underestimated, in fact the cost of RFID devices for such applications is, after all, quite low; just think that now transponders price varies from a few cents to a few dollars depending on the amount of memory or the complexity of the integrated circuit. Thus, a system conceived in this way is capable to combine an information procedures streamlining with a cost containment policy.

Ultimately, the system can operate in a simple and efficient data flow (medical records, personal data) that is immediately available and always updated, providing a definite advantage for both the medical staff and patients.

\section{References}

[1] Rossi A., Mori A., Ricci F., Maceratini R. (2001) Manuale di informatica medica, ISBN 8876205756.

[2] Giorgio A. (2011) Telemedicine: techniques and applications, In Tech Editor, ISBN 979-953-307-008-6.

[3] Vaughn W.R. (2004) Mondadori informatica (CD Rom). 